This is the accepted version of the article:

Avci C., Imaz I., Carné-Sánchez A., Pariente J.A., Tasios N., Pérez-Carvajal J., Alonso M.I., Blanco A., Dijkstra M., López C., Maspoch D.. Self-assembly of polyhedral metal-organic framework particles into three-dimensional ordered superstructures. Nature Chemistry, (2018). 10. : 78 - . 10.1038/NCHEM. 2875.

Available at: https://dx.doi.org/10.1038/NCHEM.2875 


\section{Self-Assembly of Polyhedral Metal-Organic Framework Particles into Three-Dimensional Ordered Superstructures}

Civan Avci, ${ }^{1}$ Inhar Imaz, ${ }^{1}$ Arnau Carné-Sánchez, ${ }^{1}$ Jose Angel Pariente,${ }^{2}$ Nikos Tasios, ${ }^{3}$ Javier Pérez-Carvajal, ${ }^{1}$ Maria Isabel Alonso, ${ }^{4}$ Alvaro Blanco, ${ }^{2}$ Marjolein Dijkstra, ${ }^{3}$ Cefe Lopez, ${ }^{2 *}$ and Daniel Maspoch ${ }^{1,5^{*}}$

${ }^{1}$ Catalan Institute of Nanoscience and Nanotechnology (ICN2), CSIC and The Barcelona Institute of Science and Technology, Campus UAB, Bellaterra, 08193 Barcelona, Spain

${ }^{2}$ Materials Science Factory, Instituto de Ciencia de Materiales de Madrid (ICMM), Consejo Superior de Investigaciones Científicas (CSIC), Calle Sor Juana Inés de la Cruz, 3, 28049 Madrid, Spain

${ }^{3}$ Soft Condensed Matter, Debye Institute for Nanomaterials Science, Utrecht University, Princetonplein 5, 3584 CC Utrecht, the Netherlands

${ }^{4}$ Institut de Ciència de Materials de Barcelona (ICMAB-CSIC), Campus UAB, 08193 Bellaterra, Spain

${ }^{5}$ ICREA, Pg. Lluís Companys 23, 08010 Barcelona, Spain

*Correspondence to: c.lopez@csic.es, daniel.maspoch@icn2.cat 
Self-assembly of particles into long-range, three-dimensional, ordered superstructures is crucial for the bottom-up design of plasmonic sensing materials, energy or gas storage systems, catalysts, and photonic crystals. Here we show with experimental and simulation data that truncated rhombic dodecahedral particles of the metal-organic framework (MOF) ZIF-8 can self-assemble into millimetre-sized superstructures with an underlying three-dimensional, densest rhombohedral lattice that present photonic crystal properties. We demonstrate that the photonic band gap opened in these superstructures can be tuned by controlling the size of the ZIF-8 particles. This band gap is also susceptible to the adsorption of guest substances in the micropores of the ZIF-8 particles. In addition, we demonstrate that self-assembled arrangements with different lattices could also be fabricated by tuning the truncation of ZIF-8 particles or by using octahedral UiO-66 MOF particles. These ordered, sub-micrometre featuresized superstructures made of self-assembled, porous particles might ultimately facilitate the design of novel three-dimensional photonic materials for sensing. 
Spherical colloidal particles (mainly silica or polymers such as polystyrene and acrylates) have traditionally been used to build self-assembled superstructures. ${ }^{1-4}$ They tend to pack into the entropically favoured face-centred cubic (fcc) arrangement, although they can also adopt the less favoured, hexagonal close-packed (hcp) structure or the random, hexagonally closepacked (rhcp) lattice. Recently, the self-assembly of non-spherical polyhedral particles has begun to be considered as a viable method to diversify possible packing geometries, ${ }^{5-10}$ including the formation of crystals, liquid crystals, plastic crystals and quasicrystals. ${ }^{8,11,12}$ Presently, these particles (mainly, metallic and metal chalcogenide particles such as gold, ${ }^{13,14}$ silver, ${ }^{15,16}$ cadmium/lead selenide/sulphide, ${ }^{17-19}$ and manganese oxide $)^{20}$ can be synthesised in multiple shapes, with high monodispersity and good colloidal stability, and can be coated with multiple organic polymers. Accordingly, several groups observed both experimentally and through simulations that polyhedral particles form long-range ordered assemblies, corresponding to their densest packing, by alignment of the particle facets in order to maximize the entropy of the system. ${ }^{9,12,14,15,21,22}$

To date, only a few studies have illustrated the use of polyhedral particles to generate long-range ordered arrangements that pack into different lattices. For example, Yang et al. showed that polyvinylpyrrolidone-coated cubic, octahedral and truncated octahedral silver particles (size: 100-300 nm) could be self-assembled by gravitational sedimentation into their corresponding densest lattice packings: the cubic, space-filling Kelvin structure or Minkowski packings, respectively. ${ }^{15}$ Chen, Wang et al. demonstrated that, depending on the shape, cetyltrimethylammonium bromide (CTAB)-coated gold nanoparticles can selfassemble by water evaporation into different lattices. ${ }^{13}$ They described self-assembly of gold rods, polyhedra, cubes and bipyramids into nematic/smectic-A, hexagonally-packed, tetragonally-packed, and nematic/three-dimensionally ordered arrangements. More recently, Glotzer, Lee, Mirkin et al. showed that depletion-based assembly allows 
cetyltrimethylammonium chloride (CTAC) or CTAB-coated gold nanoparticles with different shapes (rhombic dodecahedra, truncated cubes, and octahedra) to self-assemble into multiple lattices (fcc, simple cubic and body-centred cubic, respectively) that are stable both in solution and in solid state. ${ }^{14}$ Strangely, the rapid progress in the self-assembly of inorganicbased polyhedral particles has not led to the use of other families of purely organic or hybrid metal-organic materials that are usually found as crystalline polyhedral particles, as functional building blocks to construct long-range self-assembled superstructures. The use of such crystalline particles has been precluded mainly by the limited control over monodispersity, particle-shape homogeneity, and colloidal stability. Here we report that colloidal crystalline polyhedral MOF [in this case, Zeolitic Imidazolate Framework-8 (ZIF$8)^{23,24}$ and Universitetet i Oslo-66 (UiO-66 $)^{25}$ ] particles can be synthesized with the required size and shape homogeneity to be subsequently self-assembled into well-ordered threedimensional superstructures. We anticipate that these MOF-based superstructures can show new functions such as behaving as photonic crystals, in which their band gap not only can be tuned by controlling the size of the MOF particles but also is responsive to the sorption of guest substances.

\section{Results and Discussion}

Synthesis of truncated rhombic dodecahedral ZIF-8 particles. Highly monodisperse submicrometre-sized colloidal truncated rhombic dodecahedral (TRD) ZIF-8 particles in water were fabricated and stabilized by using $\mathrm{CTAB}$ as a cationic surfactant and a capping agent (Fig. 1a,b). ZIF-8 is a porous MOF made of $\mathrm{Zn}(\mathrm{II})$ ions and 2-methylimidazolate (2-MiM) linkers that exhibits a sodalite-type structure, a large surface area $\left(\approx 1200-1500 \mathrm{~m}^{2} / \mathrm{g}\right)$, good chemical and thermal stability and is apt for many interesting porosity-related applications 
(e.g. gas storage, separation, and catalysis). ${ }^{23,24,26} \mathrm{ZIF}-8$ first crystallises into cubic-shaped seeds, which gradually evolve into TRD particles, and finally, into the thermodynamically more stable rhombic dodecahedral (RD) particles, due to the predominant (100) growth. ${ }^{27,28}$ In this process, CTAB facilitates the synthesis of TRD ZIF-8 particles, as it selectively attaches to the $\{100\}$ facets (vide supra) ${ }^{29} \mathrm{By}$ controlling the amount of CTAB and 2-MiM, well-defined TRD ZIF-8 particles with sizes $(\phi$, defined as the distance between opposing square facets) from $178 \pm 8 \mathrm{~nm}$ to $227 \pm 10 \mathrm{~nm}$ were synthesized (Fig. 1c,d and Table 1). In all cases, their size dispersity was $c a$. 5\%. The TRD ZIF-8 particles exhibit six $\{100\}$ square facets with edge length $x$ (Fig. 1a and Table 1) and twelve $\{110\}$ irregular hexagonal facets showing a surface area ratio of $\sim 1: 1.5(\{100\}:\{110\})$. These TRD particles, which can also be viewed as chamfered cubes (i.e. cubes with bevelled edges), have a chamfer $(w)$ that increases with $\phi$ and a constant truncation $(t)$ of 0.69 (Table 1). X-ray powder diffraction (XRPD) indicated that all particles are pure crystalline ZIF-8 (Supplementary Fig. 1). Nitrogen physical sorption measurements on the ZIF-8 particles validated their microporosity. The measured Brunauer-Emmett-Teller (BET) surface area $\left(1154 \mathrm{~m}^{2} / \mathrm{g}\right)$ is fully consistent with previously reported values for ZIF- $8^{23}$ (Supplementary Fig. 2) and can be accounted for by internal porosity, as the external surface of the ZIF-8 is negligible in comparison.

\section{- Insert Figure 1-}

- Insert Table 1-

Self-assembly of TRD ZIF-8 particles. In the self-assembly experiments, TRD ZIF-8 particles arranged into millimetre-sized three-dimensional superstructures by evaporation 
under heating. We began by placing a droplet of an aqueous colloidal solution of the particles $(100 \mathrm{mg} / \mathrm{mL})$ on a clean glass surface. Then, the surface was incubated in an oven at $65^{\circ} \mathrm{C}$ until the droplet was dried. Field-emission scanning electron microscopy (FE-SEM) of the resulting dried solids revealed the formation of self-assembled superstructures in which the ZIF-8 particles are ordered along the three directions of space (Fig. 2 and Supplementary Fig. 3). The initial optimised evaporation temperature to ensure homogeneous $3 \mathrm{D}$ ordering along the entire monolithic structure was found to be $c a .65^{\circ} \mathrm{C}$. Evaporation under room temperature yielded 3D-ordering but led to heterogeneity in the monolith thickness, by generating thick surrounding walls around the droplet, which were formed by a concave meniscus formed over time (Supplementary Fig. 4). In contrast, a higher temperature (100 ${ }^{\circ} \mathrm{C}$ ) led to quasi-amorphous packings caused by faster evaporation (Supplementary Fig. 4). Interestingly, other tested techniques, such as dip- and-spin-coating, vertical deposition and even simple centrifugation, all yielded similar self-assembled superstructures. However, in these cases the observed degree of 3D ordering was much lower (Supplementary Figs. 4 and $5)$.

\section{- Insert Figure 2-}

Upon self-assembly, the TRD ZIF-8 particles adopted the densest rhombohedral packing, with a packing fraction of 0.86 (Figs. 2 and 3a). The same packing was also found to form in Floppy-Box Monte Carlo simulations, which is based on a simulating-annealing approach and in which the densest crystal structure is predicted by slowly increasing the pressure (Fig. 3b)..$^{30,31}$ The packing that we observed resulted from maximizing the density of the TRD particles. As shown in Figure 3c-f, the maximum number of neighbours that could 
be adjacent to a given TRD particle is twelve (Fig. 3f). Of these neighbours, six are perfectly aligned through hexagonal $\{110\}$ facets (in two groups of three that meet at two diagonally opposing vertexes; Fig. 3c), whereas the remaining six are connected through square $\{100\}$ facets (Fig. 3d). In these latter connections, the $\{100\}$ facets of neighbouring particles are touching and aligned but with a small shift (equal to $w$ ) in the two directions defined by the sides of the square (Fig. 3e). The resulting structure exhibits a rhombohedral Bravais lattice with a unit cell having the lattice parameters $\vec{a}, \vec{b}$ and $\vec{c}$ and the angles $\gamma, \beta$ and $\alpha$ as described in Table 1.

\section{- Insert Figure 3-}

Photonic crystal properties. Self-assembled spherical colloidal particles (mainly silica, polystyrene and acrylates) can form photonic crystals because they tend to order into superstructures capable of reflecting light at specific wavelengths and directions (photonic band gap) due to the periodic refractive index distribution they create. ${ }^{2,3}$ Equally, the MOFbased superstructures introduced here are periodic dielectrics made of $\approx 200-\mathrm{nm}$ MOF particles that also show angle-dependent opalescence arising from a photonic band gap. Moreover, because the lattice period is comparable to visible wavelengths, this opalescence is visible to the naked eye (Fig. 4a). Figure 4b shows spectra recorded for the superstructures assembled from TRD ZIF-8 particles of different sizes. These spectra were measured using reflectance spectrophotometry at normal incidence $\left(\theta=0^{\circ}\right)$. A clear band in the optical reflectance spectrum was observed that is attributed to the opening of a band gap in the (001) direction in reciprocal space, which is associated to the Bragg reflection from the (001) crystallographic planes of the rhombohedral lattice. Notice that these layers are not parallel to 
the square facet of the particles but form an angle given by $\cos \delta=(001) \cdot \vec{a} \times \vec{b} /|\vec{a}||\vec{b}|=\left(\phi^{2}-\right.$ $\left.w^{2}\right) /\left(\phi^{2}+2 w^{2}\right)$. The magnitude of reflectance ranged from $40 \%$ to $57 \%$ (Table 1 and Supplementary Fig. 6), which is comparable with standard literature values for selfassembled three-dimensional photonic crystals made of spherical particles. ${ }^{3}$ The reflectance spectra also showed that, firstly, the Bragg reflection spectral position scales with the particle size, and secondly, as we expected, the Bragg reflection shifted linearly towards shorter wavelengths at smaller particle sizes.

\section{- Insert Figure 4-}

Figure $4 \mathrm{c}$ shows the Bragg reflection maximum or band gap centre $\left(\lambda_{c}\right)$ plotted against the interplanar distance $(d)$. Here, it is possible to fit $\lambda_{\mathrm{c}}$ using the Bragg-Snell law for normal incidence that is defined as $\lambda_{c}=2 n d$, where $n$ is the effective index of refraction of the superstructure. For our rhombohedral superstructure, we calculated the interplanar distances by taking the normalised cross product of the lattice vectors $\vec{a}$ and $\vec{b}$, which gives the normal to the plane defined by $\vec{a}$ and $\vec{b}$ (Table 1 ). Thus, from the slope of the fitted curve, we determined an effective refractive index of 1.49 . This value is very close to the value (1.47) calculated by averaging the dielectric constant $\varepsilon=V_{\text {fram }}\left(n_{\text {fram }}\right)^{2}+V_{\mathrm{H} 2 \mathrm{O}}\left(n_{\mathrm{H} 2 \mathrm{O}}\right)^{2}+V_{\text {air }}\left(n_{\text {air }}\right)^{2}$, where $V$ and $n$ are the volume fractions and effective refractive indexes of the different components (Supplementary Section 2 and Supplementary Table 1). Our value of 1.49 is also in good agreement with the value (1.43) obtained by spectroscopic ellipsometry performed on the photonic crystal self-assembled from 210-nm TRD ZIF-8 particles (Supplementary Fig. 7). ${ }^{32}$ Importantly, the closely linear relationship between the interplanar distance (or particle size) and the maximum reflection wavelength (Fig. 4c) is a clear indication that the product 
packing fraction times average refractive index remains constant throughout the explored particle size range. That the refractive index of the MOF does not vary should not be surprising, given that all TRD ZIF-8 particles are synthesised under the same conditions. However, only particles for which the proportions are preserved, assemble into structures with the same packing fraction as this is determined by the $w / x$ ratio. As observed in Table 1 , $w$ very closely follows a linear relationship with $x$ (Supplementary Fig. 8). This indicates that the self-assembly proceeds similarly in all cases and is consistent with the fact that all the particles in the studied size range have the same geometry.

The microporosity of MOFs is highly favourable for the development of sensing applications with these photonic structures because the adsorption of species in the MOF pore network can change their refractive index, resulting in a pronounced shift in the photonic band gap spectral position, $\lambda_{\mathrm{c}}$. To realize this, the porous MOF particles should be activated, by driving out the water of the ZIF-8 particles, without disrupting the well-ordered assembly, so as to allow the adsorption of analyte molecules inside the empty pores. To this end, we studied the evacuation of the guest water molecules of the photonic structure self-assembled from 210 -nm TRD ZIF-8 particles by heating it at $260{ }^{\circ} \mathrm{C}$, a temperature at which the thermogravimetric analysis (TGA) assured the complete removal of water molecules (Supplementary Fig. 9). Indeed, TGA of the heated superstructure indicated that no further weight loss had occurred up to $300{ }^{\circ} \mathrm{C}$ (Supplementary Fig. 9), confirming that all guest water molecules had been evacuated. Moreover, FE-SEM images showed the preservation of the rhombohedral packing, whereas an $\mathrm{N}_{2}$ isotherm illustrated the typical type-I behaviour consistent with a BET surface area of $1250 \mathrm{~m}^{2} / \mathrm{g}$, thus confirming the microporous character of the photonic structure (Supplementary Figs. 10,11). Naturally, in this activated (fully dehydrated) photonic crystal the $\lambda_{\mathrm{c}}$ displaced to shorter wavelengths (from $552 \mathrm{~nm}$ to 522 $\mathrm{nm}$ ), which we expected for the replacement of water molecules with air (Supplementary Fig. 
12). As proof of concept, we subsequently exposed the activated photonic crystal to the vapour of different alcohols (methanol, ethanol, isopropanol, and $n$-butanol), and to water vapour, while measuring the shift in the photonic band gap. We found a redshift in the $\lambda_{c}$ when the photonic crystal was exposed to alcohol vapour (methanol, $\lambda_{\mathrm{c}}=561 \mathrm{~nm}$; ethanol, $\lambda_{\mathrm{c}}$ $=562 \mathrm{~nm}$; isopropanol, $\lambda_{\mathrm{c}}=564 \mathrm{~nm}$; and $n$-butanol, $\lambda_{\mathrm{c}}=568 \mathrm{~nm}$; Supplementary Figs. 13 , 14) but not when it was exposed to water. This selectivity is somewhat expected for the inherent selective adsorption properties of ZIF-8, which is known to absorb alcohols but not water. ${ }^{33}$ Considering a pore filling of $81 \%$ for methanol, ethanol or isopropanol, and $86 \%$ for $n$-butanol (calculated from the adsorption isotherms; Supplementary Fig. 15), we concluded that these shifts correlate well with those calculated according to the corresponding effective refractive indexes (determined by averaging the dielectric constant; see Supplementary Section 2 and Supplementary Table 1). Thus, the replacement of water molecules with air in the pores of ZIF-8 at relatively low pressures changes the refractive index of the photonic crystal, causing the band gap to shift. We envision that this feature could be exploited for the use of these materials as responsive materials or sensors.

Extending the self-assembly to MOF particles with other morphologies. Having demonstrated that well-ordered arrays can be fabricated from TRD ZIF-8 particles, we considered that the numerous MOFs reported to date could be an excellent source of crystalline polyhedral particles. We inferred that control of the particle size, shape and selfassembly could enable new packing geometries. To this end, we extended the concept of using MOF particles to spontaneously generate ordered arrays by using ZIF-8 particles with other morphologies (i.e. TRD with a lower degree of truncation, and non-truncated RD), as well as octahedral particles of the UiO-66 MOF (Fig. 5). For the synthesis of TRD ZIF-8 particles with less truncation $(\phi=263 \pm 13 \mathrm{~nm} ; x=107 \pm 5 \mathrm{~nm} ; w=78 \pm 4 \mathrm{~nm} ; t=0.57 v s$. 0.69 of the previously synthesised TRD ZIF-8 particles), the capping effect of CTAB was 
used again; however, the amount of CTAB was reduced to accelerate the growth of the $\{100\}$ facets (Supplementary Fig. 17). Complete removal of the CTAB resulted in either slightly truncated $(\phi=319 \pm 14 \mathrm{~nm} ; x=76 \pm 3 \mathrm{~nm} ; w=122 \pm 5 \mathrm{~nm} ; t=0.38$; Supplementary Fig. 17) or non-truncated $(t=0)$ RD ZIF-8 particles, depending on the reaction time. The latter particles exhibited a $\phi$ (defined as the distance between opposing vertices) of $530 \pm 30 \mathrm{~nm}$ and an edge size of $230 \pm 12 \mathrm{~nm}$ (Supplementary Fig. 17).

\section{- Insert Figure 5-}

Experimental observations confirmed that the new ZIF-8 particles self-assemble into ordered arrangements once their colloidal solutions are evaporated off at either $65{ }^{\circ} \mathrm{C}$ (TRD particles) or $120{ }^{\circ} \mathrm{C}$ (RD particles). A theoretical analysis of the densest packings as a function of $t$ of the ZIF-8 particles is shown in Figure 5a. Here we found that the densest packing is a rhombohedral crystal for TRD particles with a truncation $t$ higher than 0.66 , whereas it is an fcc crystal for $t$ lower than 0.66 . These theoretical findings corroborate the experimentally obtained self-assembled superstructures. As shown above, monodisperse TRD ZIF-8 particles with $t=0.69$ adopted the rhombohedral packing, whereas the newly synthesised particles with $t=0.57$ crystallized into a plastic fcc crystal with a packing fraction of 0.68 (Fig. 5b). Note that the plastic fcc crystal differs from the regular one in the sense that no preferential facet-to-facet interactions exist between neighbouring ZIF-8 particles. Here, we reasoned that the formation of a plastic -rather than a regular- fcc crystal was due to kinetic effects. Consistent with the simulation data, the slightly truncated ZIF-8 particles $(t=0.38)$ and the RD ZIF-8 particles $(t=0)$ each crystallized into an fcc regular crystal (Fig. 5c). In the latter crystals, each ZIF-8 particle is bound to twelve other particles 
by their twelve identical $\{110\}$ facets. Importantly, the self-assembly experiments that we ran with as-synthesised, CTAB-free ZIF-8 particles all failed to produce ordered packings, which can be explained by the strong Van der Waals attractions between the particles that are not balanced by ionic surfactants (like CTAB) on the surface of these particles. This result parallels observations on the assembly of polyhedral inorganic particles, in which adsorbed ionic surfactants/polymers on the particle surfaces is crucial to stabilise the suspension against irreversible colloidal aggregation. ${ }^{14,15}$ We wish to remark here that non-adsorbed ionic surfactants/polymers in solution can be used to assist the self-assembly by inducing depletion attractions between the particles.

Finally, we extended our approach to another MOF: we synthesised monodisperse octahedral particles of UiO- $66^{25}(\phi$, defined as the distance between opposing vertices: $342 \pm$ $30 \mathrm{~nm}$; edge size: $242 \pm 18 \mathrm{~nm}$ ) using acetic acid as a coordination modulator agent (Supplementary Fig. 17). ${ }^{34} \mathrm{UiO}-66$ is a promising MOF for catalysis and $\mathrm{CO}_{2}$ capture, owing to its large surface area $\left(1100-1400 \mathrm{~m}^{2} / \mathrm{g}\right)$ and to its high hydrothermal, chemical and thermal stability. ${ }^{35,36}$ After the synthesis, we functionalized the UiO-66 particles with CTAB and, using the evaporation method at $85^{\circ} \mathrm{C}$, left them to self-assemble. FESEM images revealed the formation of an ordered arrangement showing a hexagonal lattice closely related to the Minkowski lattice (Fig. 5d), as has been observed for octahedral silver particles. ${ }^{15}$

\section{Conclusions}

We have demonstrated that crystalline, polyhedral TRD ZIF-8 particles can be synthesised with good monodispersity, shape homogeneity, and colloidal stability and that they subsequently (spontaneously) self-assemble into millimetre-sized three-dimensional ordered arrangements. The resulting superstructures, corresponding to the densest rhombohedral 
packings, are porous and show a photonic band gap functionality that can be tuned by controlling the size of the ZIF-8 particles or by changing the substance adsorbed in the micropores of the ZIF-8 particles. These characteristics should enable the development of self-assembled, MOF-based photonic crystals for sensing applications. In addition, we have extended the formation of ordered arrangements showing different packing geometries to include the self-assembly of crystalline MOF particles with other shapes. This first evidence confirms that MOF particles and other molecular crystalline particles, like purely organic particles, can be harnessed for the self-assembly of superstructures with long-range periodicities, thereby opening up new avenues for the design of novel materials for applications as sensing, storage, catalysis, and photonics.

\section{Methods}

Synthesis of TRD ZIF-8 particles with truncation $\boldsymbol{t}=\mathbf{0 . 6 9}$ or 0.57 . In a typical synthesis, 5 $\mathrm{mL}$ of an aqueous solution of $\mathrm{Zn}\left(\mathrm{CH}_{3} \mathrm{COO}\right)_{2} \cdot 2 \mathrm{H}_{2} \mathrm{O}(300 \mathrm{mg})$ was added to $5 \mathrm{~mL}$ of an aqueous solution containing varying amounts of 2-methylimidazole (2-MiM) and CTAB ${ }^{29}$ with gentle stirring for a few seconds. The resulting transparent mixture turned white after 15 $\mathrm{s}$ and was left undisturbed at room temperature for $2 \mathrm{~h}$. The resulting ZIF-8 particles were washed three times with DI water upon centrifugation at $9000 \mathrm{rpm}$ in $50-\mathrm{mL}$ Falcon tubes. The collected wet pellets were finally re-dispersed in DI water at a concentration of 100 $\mathrm{mg} / \mathrm{mL}$. Note that in order to prevent aggregation, the particles should be dispersed while they are still wet. The zeta potential of the resulting TRD ZIF-8 particles was $c a .+40 \mathrm{mV}$. The conditions used to synthesise the TRD ZIF-8 particles with $t=0.69$ of each size were: for $178 \pm 8 \mathrm{~nm},[\mathrm{CTAB}]=0.54 \mathrm{mM}$ and $[2-\mathrm{MiM}]=2.72 \mathrm{M}$; for $193 \pm 8 \mathrm{~nm},[\mathrm{CTAB}]=0.5$ $\mathrm{mM}$ and $[2-\mathrm{MiM}]=2.72 \mathrm{M}$; for $210 \pm 10 \mathrm{~nm},[\mathrm{CTAB}]=0.54 \mathrm{mM}$ and $[2-\mathrm{MiM}]=2.58 \mathrm{M}$; 
and for $227 \pm 10 \mathrm{~nm},[\mathrm{CTAB}]=0.44 \mathrm{mM}$ and $[2-\mathrm{MiM}]=2.72 \mathrm{M}$. The conditions used to synthesise the TRD ZIF-8 particles with $t=0.57$ were: $[\mathrm{CTAB}]=0.14 \mathrm{mM}$ and $[2-\mathrm{MiM}]=$ 3.81 M.

Synthesis of TRD ZIF-8 particles with truncation $t=0.38$, and RD ZIF-8 particles: $5 \mathrm{~mL}$ of an aqueous solution of $\mathrm{Zn}\left(\mathrm{CH}_{3} \mathrm{COO}\right)_{2} \cdot 2 \mathrm{H}_{2} \mathrm{O}(300 \mathrm{mg})$ were added to $6.4 \mathrm{~mL}$ of an aqueous solution of $2.72 \mathrm{M}$ 2-MiM with gentle stirring. After $15 \mathrm{~s}$ the transparent mixture turned white, and was then left undisturbed at room temperature for either 15 min (TRD ZIF8 particles with $t=0.38$ ) or $2 \mathrm{~h}$ (RD ZIF-8 particles). The resulting ZIF-8 particles were washed three times with DI water with centrifugation at $9000 \mathrm{rpm}$ in 50-mL Falcon tubes. For functionalization, the particles were re-dispersed in $0.54 \mathrm{mM} \mathrm{CTAB} \mathrm{(aq.),} \mathrm{stirred} \mathrm{for} 30$ min, and washed once more with DI water with centrifugation at $9000 \mathrm{rpm}$ in 50-mL Falcon tubes. The collected wet pellets were finally re-dispersed in DI water at a concentration of $100 \mathrm{mg} / \mathrm{mL}$. The zeta potential of the resulting ZIF-8 particles was $c a .+50 \mathrm{mV}$.

Synthesis of octahedral UiO-66 particles. In a typical synthesis, $\mathrm{ZrCl}_{4}(34.9 \mathrm{mg})$ and 1-4benzendicarboxylic acid (BDC; $24.9 \mathrm{mg}$ ) were dissolved in $10 \mathrm{~mL}$ of $2.1 \mathrm{M}$ acetic acid (DMF) and transferred to a scintillation vial that was placed in a pre-heated oven at $120{ }^{\circ} \mathrm{C}$ for 12 hours. The colloidal crystals were then recovered by centrifugation and washed twice with DMF and twice with methanol with centrifugation at $9000 \mathrm{rpm}$ in a 50-ml Falcon tube. For functionalization, the particles were re-dispersed in 0.54 mM CTAB (aq.), stirred for 30 min, and washed once more with DI water with centrifugation at $9000 \mathrm{rpm}$ in 50-mL Falcon tubes. The collected wet pellets were finally re-dispersed in DI water at a concentration of $100 \mathrm{mg} / \mathrm{mL}$. The zeta potential of the resulting octahedral UiO-66 particles was $c a .+50 \mathrm{mV}$.

Formation of the self-assembled superstructures. Glass microscope slides were initially washed with water and ethanol, dried with a pressurised $\mathrm{N}_{2}$ gun, and further subjected to 
plasma cleaning under $\mathrm{Ar}^{+}$for 2 min using a Zepto Plasma Cleaner (Diener Electronic). Then, $150 \mu \mathrm{L}$ of each colloidal aqueous solution of MOF particles was added dropwise onto the clean substrate, and the treated sample was left to dry in an oven at varying temperatures (T). The temperatures used to fabricate the self-assembled superstructures were: $65^{\circ} \mathrm{C}$ (TRD ZIF-8), $120^{\circ} \mathrm{C}$ (RD ZIF-8 T) or $85^{\circ} \mathrm{C}$ (octahedral UiO-66).

Floppy-Box Monte Carlo Simulations. In order to predict the densest crystal structure of hard MOF particles, we performed Floppy-Box Monte Carlo simulations. ${ }^{30,31}$ In this method, we perform constant-pressure simulations of $\mathrm{N}=1,2,3$, or 4 particles in a simulation box with a variable box shape and periodic boundary conditions. We compressed the system by increasing the pressure in stepwise, which enabled us to predict the densest packing. In all cases, we obtained the densest packing, as presented in Figs. 2 and 3.

Data availability. All the data supporting the findings of this study are available upon request from the corresponding author (including data presented in the main text and in the Supplementary Information).

\section{References}

1. Li, F., Josephson, D. P. \& Stein, A. Colloidal assembly: the road from particles to colloidal molecules and crystals. Angew. Chem. Int. Ed. 50, 360-88 (2011).

2. von Freymann, G., Kitaev, V., Lotsch, B. V \& Ozin, G. A. Bottom-up assembly of photonic crystals. Chem. Soc. Rev. 42, 2528-2554 (2013).

3. Galisteo-López, J. F. et al. Self-assembled photonic structures. Adv. Mater. 23, 30-69 (2011).

4. Kim, S.-H., Lee, S. Y., Yang, S.-M. \& Yi, G.-R. Self-assembled colloidal structures for photonics. NPG Asia Mater. 3, 25-33 (2011). 
5. Glotzer, S. C. \& Solomon, M. J. Anisotropy of building blocks and their assembly into complex structures. Nature Mater. 6, 557-562 (2007).

6. Quan, Z. \& Fang, J. Superlattices with non-spherical building blocks. Nano Today 5, 390-411 (2010).

7. Damasceno, P. F., Engel, M. \& Glotzer, S. C. Predictive self-assembly of polyhedra into complex structures. Science 337, 453-457 (2012)

8. Agarwal, U. \& Escobedo, F. A. Mesophase behaviour of polyhedral particles. Nature Mater. 10, 230-235 (2011).

9. Torquato, S. \& Jiao, Y. Dense packings of the Platonic and Archimedean solids. Nature 460, 876-879 (2009).

10. Sacanna, S. \& Pine, D. J. Shape-anisotropic colloids: Building blocks for complex assemblies. Curr. Opin. Colloid Interface Sci. 16, 96-105 (2011).11. Gantapara, A. P., de Graaf, J., van Roij, R. \& Dijkstra, M. Phase diagram and structural diversity of a family of truncated cubes: degenerate close-packed structures and vacancy-rich states. Phys. Rev. Lett. 111, 015501 (2013)

12. Haji-Akbari, A. et al. Disordered, quasicrystalline and crystalline phases of densely packed tetrahedra. Nature 462, 773-777 (2009).

13. Ming, T. et al. Ordered gold nanostructure assemblies formed by droplet evaporation. Angew. Chem. Int. Ed. 47, 9685-9690 (2008).

14. Young, K. L. et al. A Directional entropic force approach to assemble anisotropic nanoparticles into superlattices. Angew. Chem. Int. Ed. 52, 13980-13984 (2013).

15. Henzie, J., Grünwald, M., Widmer-Cooper, A., Geissler, P. L. \& Yang, P. Self-assembly of uniform polyhedral silver nanocrystals into densest packings and exotic superlattices. Nature Mater. 11, 131-137 (2012). 
16. Tao, A. R., Ceperley, D. P., Sinsermsuksakul, P., Neureuther, A. R. \& Yang, P. Self-organized silver nanoparticles for three-dimensional plasmonic crystals. Nano Lett. 8, 4033-4038 (2008).

17. Miszta, K. et al. Hierarchical self-assembly of suspended branched colloidal nanocrystals into superlattice structures. Nature Mater. 10, 872-876 (2011).

18. Geuchies, J. J. et al. In situ study of the formation mechanism of two-dimensional superlattices from PbSe nanocrystals. Nature Mater. 15, 1248-1254 (2016).

19. Boneschanscher, M. P. et al. Long-range orientation and atomic attachment of nanocrystals in 2D honeycomb superlattices. Science 344, 1377-1380 (2014).

20. Xie, S. et al. Supercrystals from crystallization of octahedral MnO nanocrystals. J. Phys. Chem. C 113, 19107-19111 (2009).

21. Volkov, N., Lyubartsev, A. \& Bergstrom, L. Phase transitions and thermodynamic properties of dense assemblies of truncated nanocubes and cuboctahedra. Nanoscale 4, 4765-4771 (2012).

22. Damasceno, P. F., Engel, M. \& Glotzer, S. C. Crystalline assemblies and densest packings of a family of truncated tetrahedra and the role of directional entropic forces. ACS Nano $\mathbf{6 , 6 0 9 -}$ 614 (2012).

23. Park, K. S. et al. Exceptional chemical and thermal stability of zeolitic imidazolate frameworks. Proc. Natl. Acad. Sci. 103, 10186-10191 (2006).

24. Huang, X.-C., Lin, Y.-Y., Zhang, J.-P. \& Chen, X.-M. Ligand-directed strategy for zeolitetype metal-organic frameworks: zinc(II) imidazolates with unusual zeolitic topologies. Angew. Chem. Int. Ed. 45, 1557-1559 (2006).

25. Cavka, J. H. et al. A new zirconium inorganic building brick forming metal organic frameworks with exceptional stability. J. Am. Chem. Soc. 130, 13850-13851 (2008).

26. Chen, B., Yang, Z., Zhu, Y. \& Xia, Y. Zeolitic imidazolate framework materials: recent 
progress in synthesis and applications. J. Mater. Chem. A 2, 16811-16831 (2014).

27. Cravillon, J. et al. Controlling zeolitic imidazolate framework nano- and microcrystal formation: insight into crystal growth by time-resolved in situ static light scattering. Chem.

Mater. 23, 2130-2141 (2011).

28. Cravillon, J. et al. Formate modulated solvothermal synthesis of ZIF-8 investigated using time-resolved in situ X-ray diffraction and scanning electron microscopy. CrystEngComm 14, 492-498 (2012).

29. Pan, Y. et al. Tuning the crystal morphology and size of zeolitic imidazolate framework-8 in aqueous solution by surfactants. CrystEngComm 13, 6937-6940 (2011).30. ～de Graaf, J., van Roij, R. \& Dijkstra, M. Dense regular packings of irregular nonconvex particles. Phys. Rev. Lett. 107, 155501 (2011).

31. Filion, L. et al. Efficient method for predicting crystal structures at finite temperature: variable box shape simulations. Phys. Rev. Lett. 103, 188302 (2009).

32. Ahles, M. et al. Spectroscopic ellipsometry on opaline photonic crystals. Optics Commun. 246, 1-7 (2005).

33. Zhang, K. et al. Alcohol and water adsorption in zeolitic imidazolate frameworks. Chem. Commun. 49, 3245-3247 (2013).

34. Andreas Schaate. et al. Modulated synthesis of Zr-based metal-organic frameworks: from nano to single crystals. Chem. Eur. J. 17, 6643-6651 (2011)

35. Vermoortele, F. et al. Synthesis modulation as a tool to increase the catalytic activity of metalorganic frameworks: the unique case of UiO-66(Zr). J. Am. Chem. Soc. 135, 11465-11468 (2013).

36. $\mathrm{Wu}, \mathrm{H}$. et al. Unusual and highly tunable missing-linker defects in zirconium metal-organic framework UiO-66 and their important effects on gas adsorption. J. Am. Chem. Soc. 135, $10525-10532(2013)$. 


\section{Acknowledgments}

This work was supported by the EU FP7 ERC-Co 615954 and the Spanish MINECO (projects PN MAT2012-30994 and MAT2015-68075-R [SIFE2]) and the Comunidad de Madrid project S2013/MIT-2740 (PHAMA_2.0). I.I. thanks the Spanish MINECO for the RyC fellowship RyC-2010-06530. The authors based at ICN2 and ICMAB acknowledge the support of the Spanish MINECO through the Severo Ochoa Centers of Excellence Program, under Grants SEV-2013-0295 and SEV-2015-0496. We also thank Jorge Albalad and Dr. Javier Saiz for their help in the TGA and reflectance measurements, respectively.

\section{Author contributions}

C.A. and I.I. synthesised the ZIF-8 particles and the corresponding self-assembled superstructures. A.C.S. synthesised the UiO-66 particles and the corresponding selfassembled superstructures. N.T. and M.D. performed the Floppy-Box Monte Carlo simulations. C.A., J.A.P., A.B. and C.L. performed and analysed the photonic measurements. M.I.A. performed the ellipsometry characterisation, and C.A. and J.P.C. performed the sorption measurements. D.M. conceived the project and drafted the manuscript. All authors discussed the results and commented on the manuscript.

\section{Additional information}

Supplementary information is linked to the online version of the paper at: www.nature.com/nchem.

\section{Competing financial interests}

The authors declare no competing financial interests. 


\section{Figure Captions}

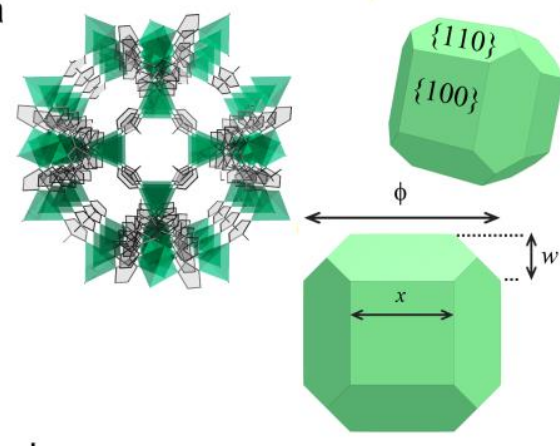

b

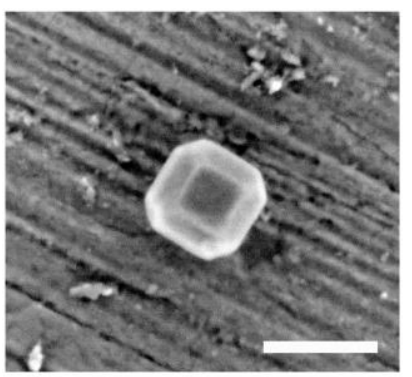

$\mathrm{C}$

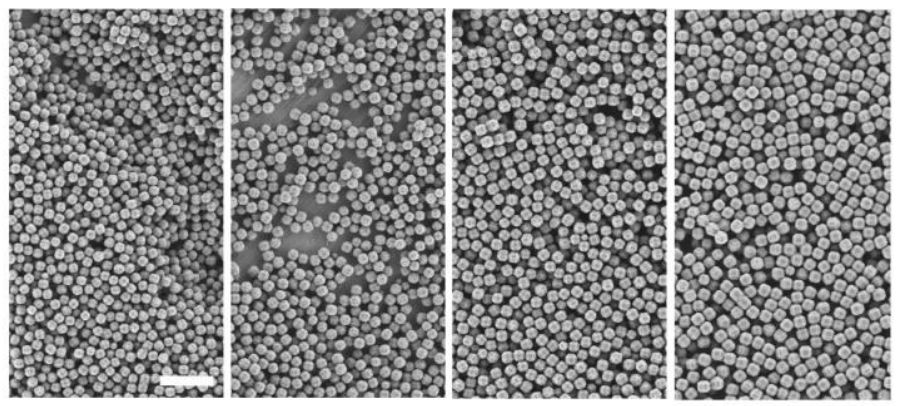

d

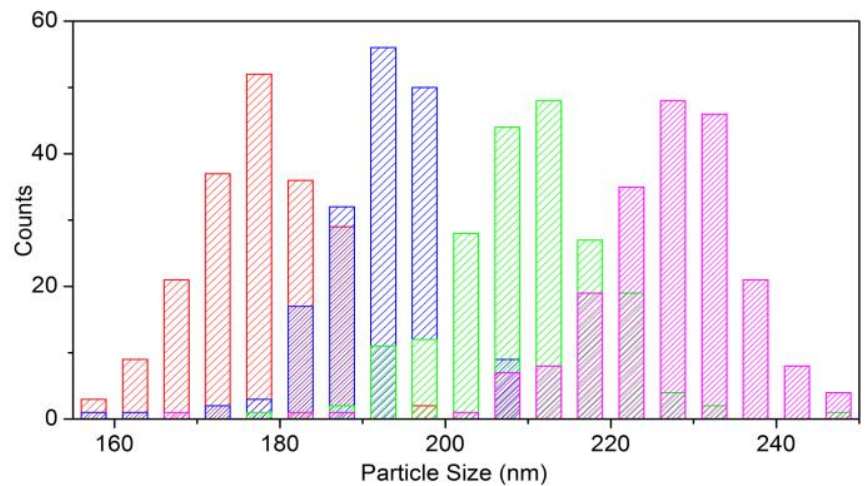

Figure 1. Formation and characterization of the TRD ZIF-8 particles. a, Representation of the crystal structure of ZIF-8 (left) and schematic illustrations of the ZIF-8 TRD particles, highlighting the particle size ( $\phi)$, the edge length $(x)$ of the $\{100\}$ square facets, and the chamfer ( $w$ ) (right). b, Representative FE-SEM image of a single TRD ZIF-8 particle. $\mathbf{c}$, Representative FE-SEM images of the TRD ZIF-8 particles of different sizes (from left to right): $178 \pm 8 \mathrm{~nm}, 193 \pm 8 \mathrm{~nm}, 210 \pm 10 \mathrm{~nm}$, and $227 \pm 10 \mathrm{~nm}$. d, Size-distribution histograms of the TRD ZIF-8 particles of different sizes: red: $178 \pm 8 \mathrm{~nm}$; blue: $193 \pm 8 \mathrm{~nm}$; green: $210 \pm 10 \mathrm{~nm}$; and purple: $227 \pm 10 \mathrm{~nm}$. Scale bars: $200 \mathrm{~nm}(\mathbf{b})$ and $1 \mu \mathrm{m}(\mathbf{c})$. 
a

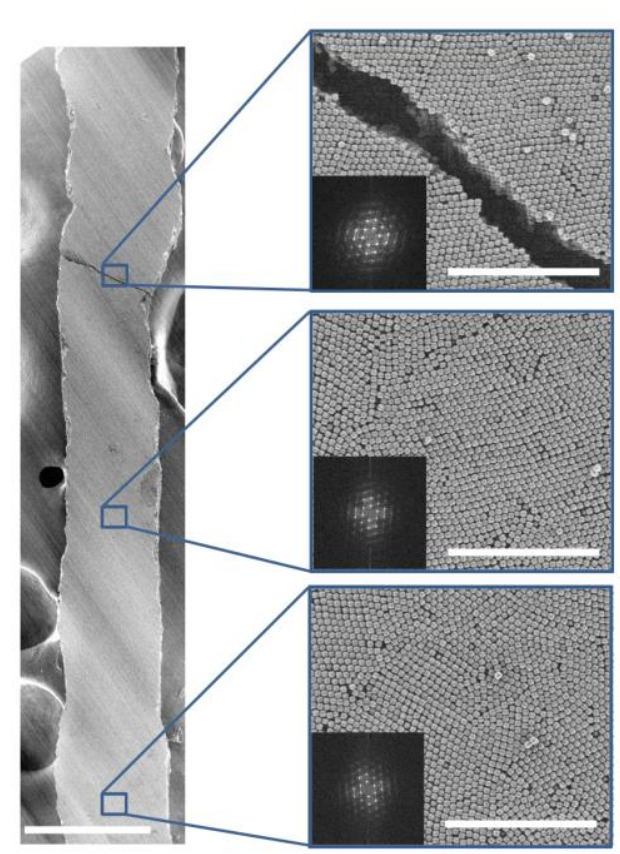

b

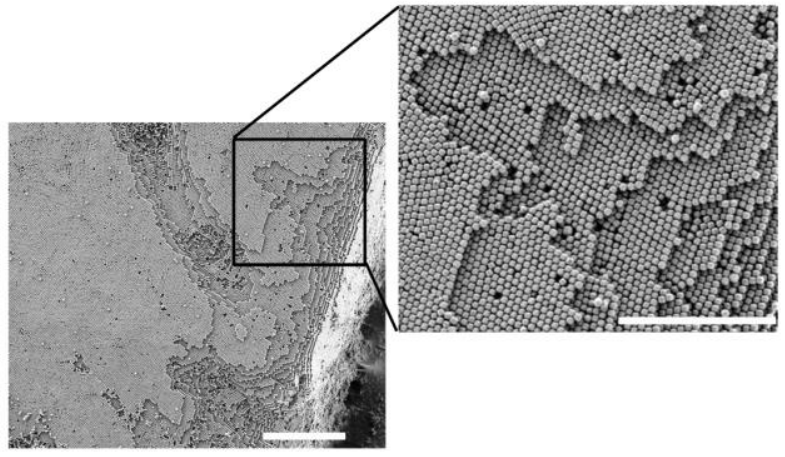

C

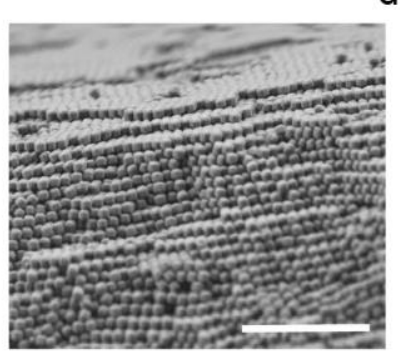

d

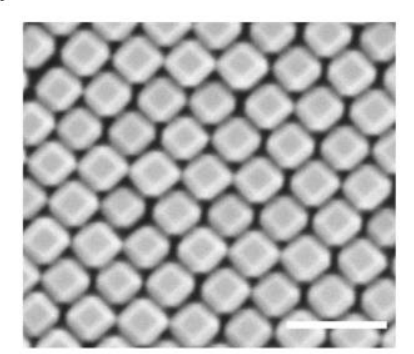

Figure 2. Ordered rhombohedral self-assembled superstructures. Representative FE-SEM images of a selfassembled superstructure made of TRD ZIF-8 crystals sized $210 \pm 10 \mathrm{~nm}$. a, Low-magnification image of a selfassembled superstructure that extends over $1 \mathrm{~mm}$. The three magnified sections reveals that the rhombohedral lattice is maintained across the self-assembled superstructure. Note here that, like in any other superstructure made of self-assembled particles, the ordering of TRD ZIF-8 crystals reveals domains with misfit dislocations and point defects. Insets: the Fourier transforms of these sections showing different grain orientations. b, Edge of a self-assembled superstructure, showing order in the three-dimensions. c, Cross-section of the selfassembled superstructure. d, Zoom on the packing structure. Scale bars: $200 \mu \mathrm{m}$ (a-left), $10 \mu \mathrm{m}(\mathbf{b}), 5 \mu \mathrm{m}(\mathbf{a}-$ right), $2 \mu \mathrm{m}(\mathbf{c}), 1 \mu \mathrm{m}(\mathbf{b}$, zoom $)$, and $500 \mathrm{~nm}(\mathbf{d})$. 
a

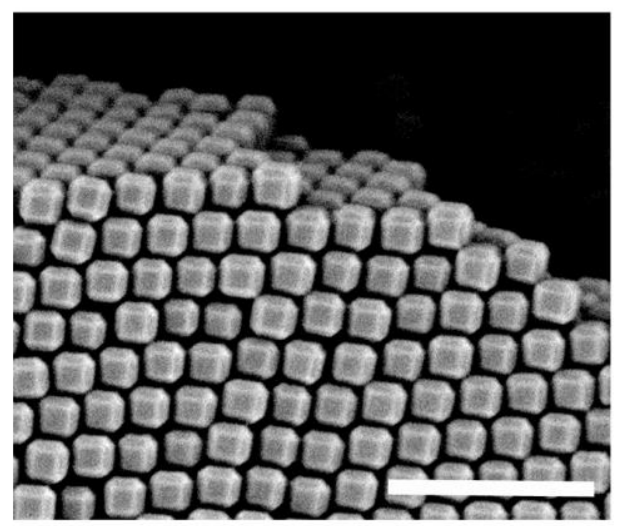

C

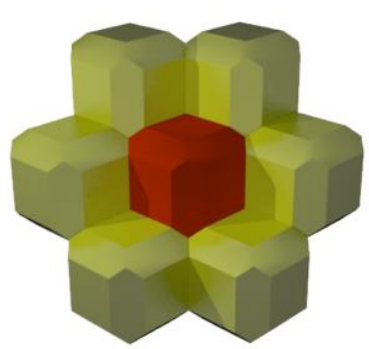

d

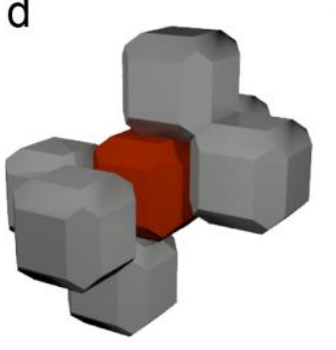

b

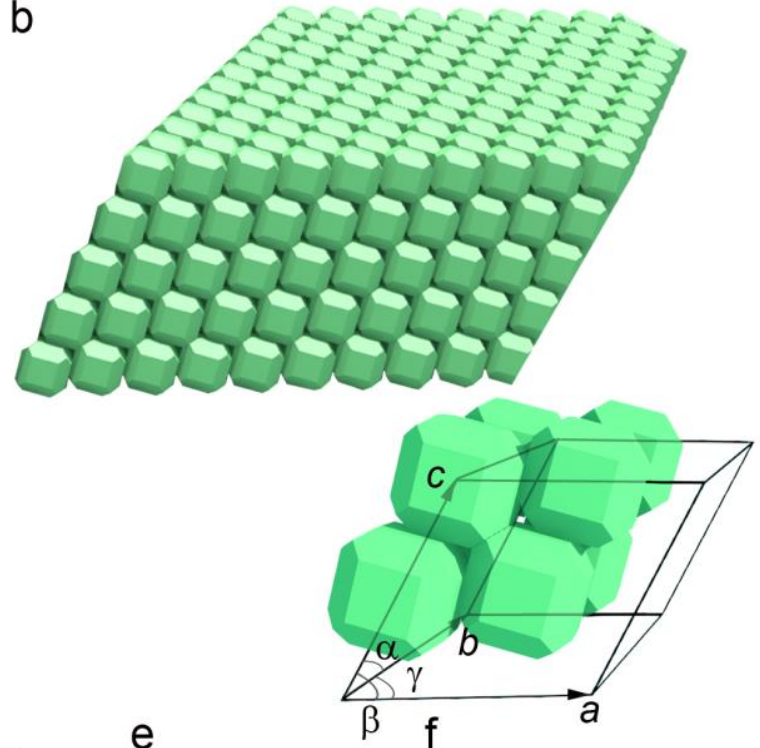

e
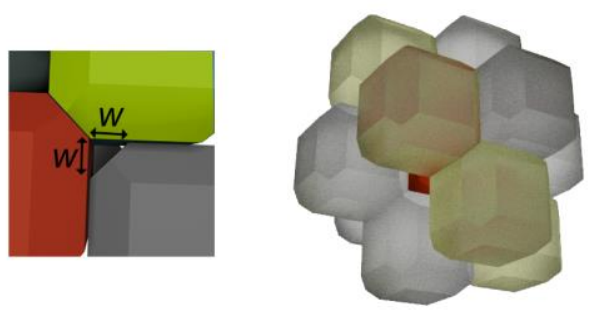

Figure 3. Computer simulation and FE-SEM image of the formation of the densest rhombohedral lattice.

a, Representative FE-SEM image of a cross-section of the self-assembled superstructure, showing the rhombohedral lattice. b, Densest packing obtained from Floppy-Box Monte Carlo simulations, confirming the same type of rhombohedral lattice obtained experimentally. Unit cell of the rhombohedral lattice (below). c-f, Schematic illustration showing the connectivity of a single TRD ZIF-8 particle in the rhombohedral lattice: six TRD particles perfectly aligned through the $\{110\}$ facets (c); six TRD particles aligned through the $\{100\}$ facets (d); representation showing the shift in two directions of these latter connections (d); combined twelve TRD particles aligned to a single TRD particle (f). Scale bar: $1 \mu \mathrm{m}(\mathbf{a})$. 
a

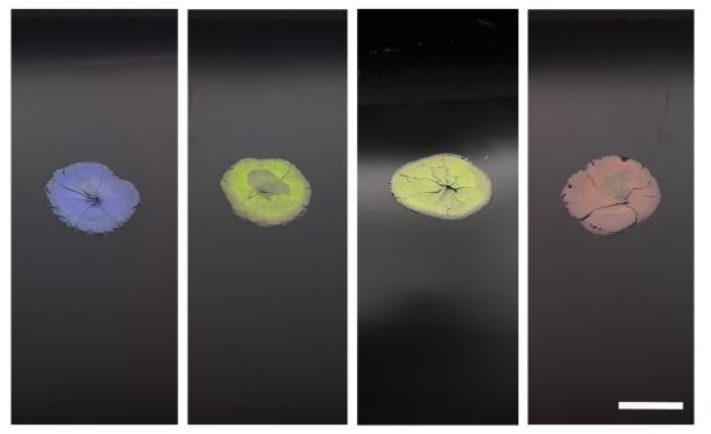

b

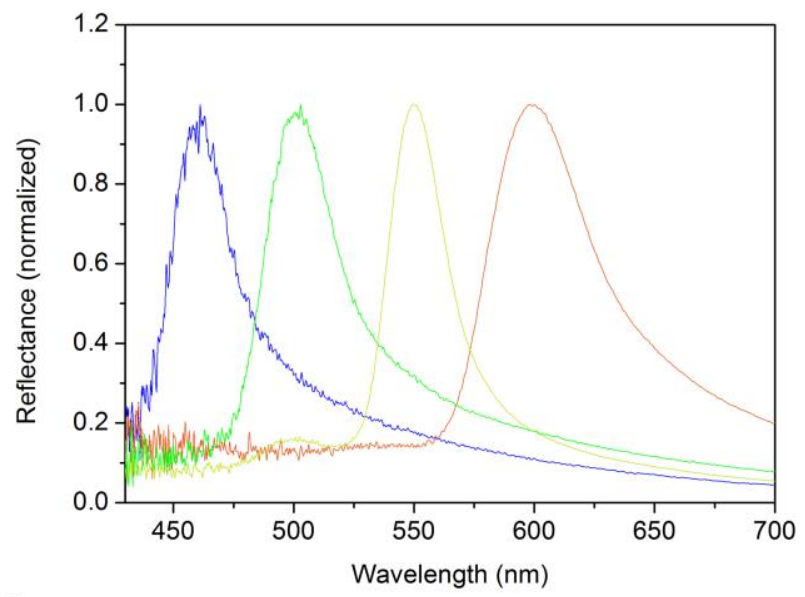

C

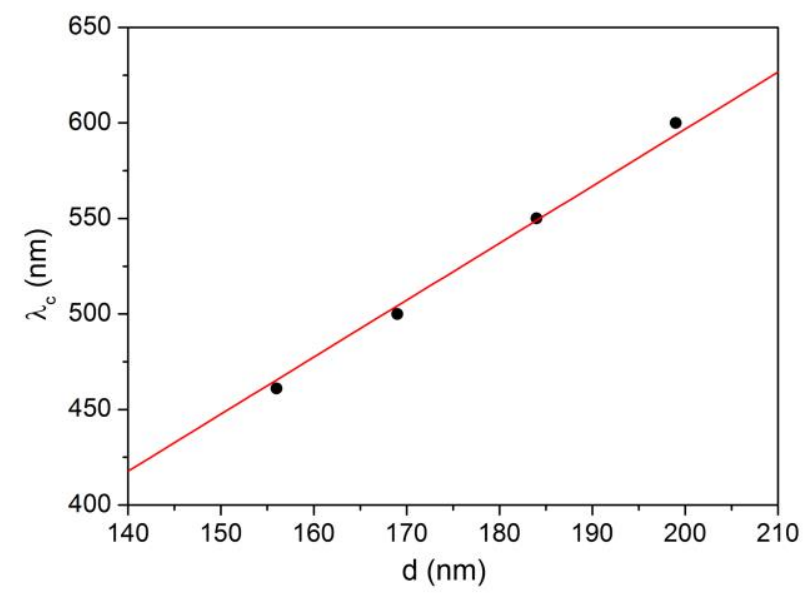

Figure 4. Photonic properties. a,b, Photographs (a) and normalised optical reflection at $\theta=0^{\circ}$ of the selfassembled photonic crystals made of TRD ZIF-8 particles of different sizes (b): $178 \pm 8 \mathrm{~nm}$ (blue); $193 \pm 8 \mathrm{~nm}$ (green); $210 \pm 10 \mathrm{~nm}$ (yellow); and $227 \pm 10 \mathrm{~nm}$ (red). c, The Bragg reflection maximum wavelength $\left(\lambda_{\mathrm{c}}\right)$ plotted against the interplanar distance (d) and fit to the Bragg law (red line) constrained to intercept at zero. Scale bar: $1 \square \mathrm{m}(\mathbf{a})$. 
a

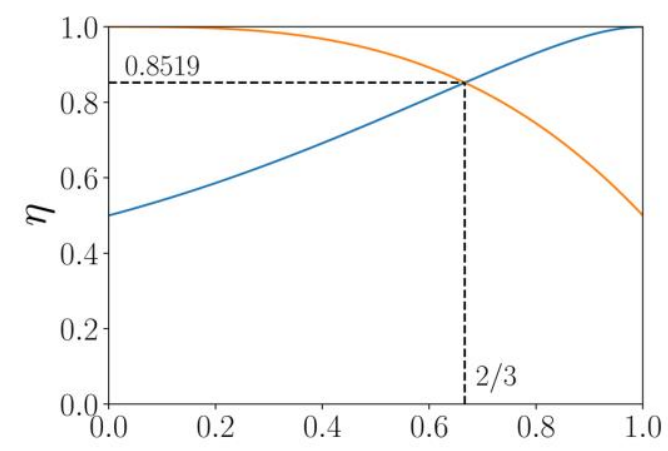

b

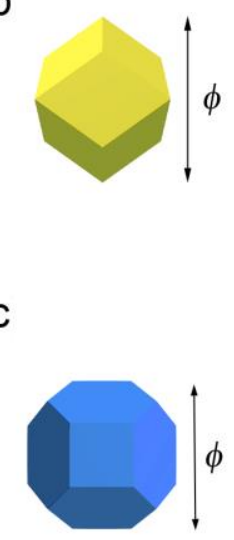

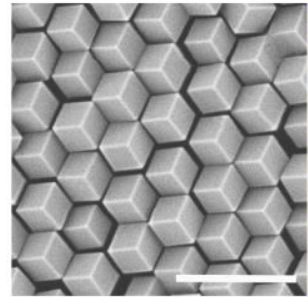

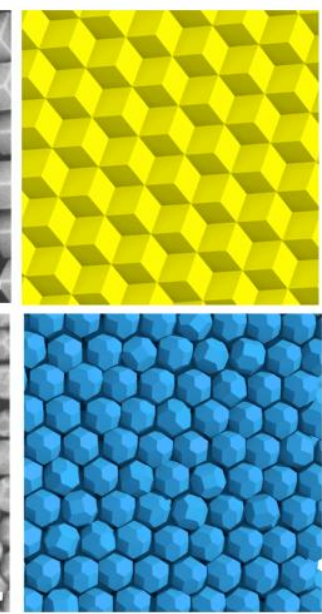

d
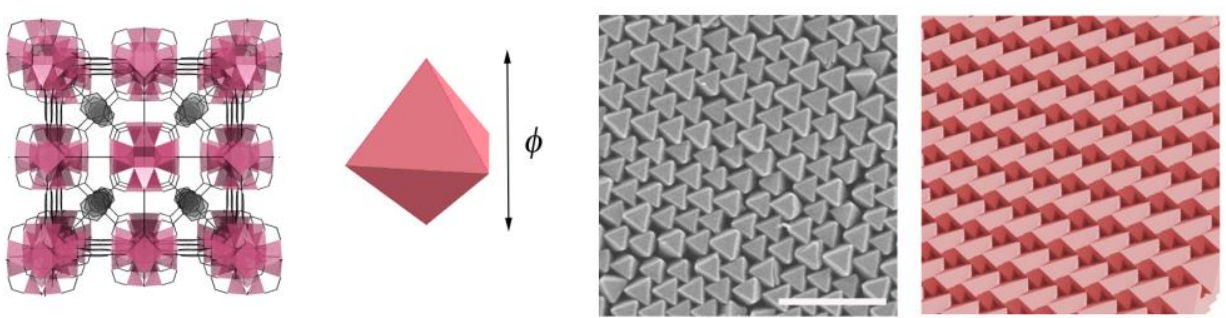

Figure 5. Ordered self-assembled superstructures made of MOF particles with other morphologies. a,

Maximum packing fraction as a function of the truncation $(t)$ of the ZIF-8 particles for the two possible packing geometries: rhombohedral (blue) and fcc (orange). b-d, (left) Schematic representation of the single MOF particles and (middle) representative FE-SEM images of the plastic fcc crystal, the regular fcc crystal and the hexagonal packing closely related to the Minkowski lattice resulting from the self-assembly of TRD ZIF- 8 particles with $\mathrm{t}=0.57(\mathbf{b}), \mathrm{RD}$ ZIF-8 particles $(\mathbf{c})$ or octahedral UiO-66 particles $(\mathbf{d})$, respectively. Note that a representation of the crystal structure of UiO-66 is also included in (d). (right) Corresponding packings obtained from Floppy-Box Monte Carlo simulations. Scale bar: $1 \mu \mathrm{m}(\mathbf{b}-\mathbf{d})$. 
Avci, C. et al., Table 1.

Table 1. Length parameters of the particles, and lattice parameters and angles, interplanar distances, optical band gaps and reflectance of the self-assembled superstructures.

\begin{tabular}{|c|c|c|c|c|c|c|c|c|}
\hline $\begin{array}{l}\text { Particle } \\
\text { size } \\
\phi(n m){ }^{\dagger}\end{array}$ & $x(\mathrm{~nm})^{\dagger}$ & $\begin{array}{c}\mathrm{w} \\
(\mathrm{nm})\end{array}$ & $t^{\S}$ & $\begin{array}{c}\text { Lattice } \\
\text { parameters } \\
\mathrm{a}, \mathrm{b}, \underset{\S \S}{\mathrm{c}}(\mathrm{nm})\end{array}$ & $\begin{array}{c}\text { Lattice } \\
\text { angles } \\
\gamma, \beta, \alpha \\
()^{0}\end{array}$ & $\begin{array}{l}\text { Interplanar } \\
\text { distance } \\
d(n m)^{*}\end{array}$ & $\begin{array}{l}\text { Band } \\
\text { gap } \\
(\mathrm{nm}) \\
+t\end{array}$ & $\begin{array}{c}\text { Reflectance } \\
(\%)^{\dagger+}\end{array}$ \\
\hline $178 \pm 8$ & $93 \pm 4$ & $43 \pm 2$ & 0.69 & 188 & 61 & 156 & 461 & 47 \\
\hline $193 \pm 8$ & $101 \pm 4$ & $46 \pm 2$ & 0.69 & 204 & 61 & 169 & 500 & 50 \\
\hline $210 \pm 10$ & $110 \pm 5$ & $50 \pm 2$ & 0.69 & 222 & 61 & 184 & 552 & 57 \\
\hline $227 \pm 10$ & $119 \pm 5$ & $54 \pm 2$ & 0.69 & 239 & 61 & 199 & 600 & 43 \\
\hline $\begin{array}{l}{ }^{\dagger} \text { Measure } \\
{ }^{\ddagger} \text { Calculat } \\
\S \text { Calculat } \\
{ }^{\S \S} \text { Calculat } \\
{ }^{\circledR} \text { Calculat } \\
{ }^{*} \text { Calculat } \\
{ }^{\dagger \dagger} \text { Measur }\end{array}$ & $\begin{array}{l}\text { from } F E- \\
\text { from }(\phi- \\
\text { from } x /(x \\
d \text { from }(2 v \\
\text { from cos } \\
d \text { from }[2 w \\
d \text { from UV }\end{array}$ & $\begin{array}{l}\text { EM imag } \\
\text { /2 } \\
-w) \\
\left.+\phi^{2}\right)^{1 / 2} \\
{\left[\left(w^{2}+2 \phi\right.\right.} \\
\left.w^{2}-\phi w\right) \\
\text { Vis reflec }\end{array}$ & $\begin{array}{l}\text { w) / }(21 \\
+\phi\left(\phi^{2}-\right. \\
\text { tance s }\end{array}$ & $\begin{array}{l}\left.\left.v^{2}+\phi^{2}\right)\right] \\
\left.\left.w^{2}\right)\right] /\left[2\left(w^{2}-\right.\right. \\
\text { pectra }\end{array}$ & $v)^{2}+\left(\phi^{2}\right.$ & $\left.\left.N^{2}\right)^{2}\right]^{1 / 2}$ & & \\
\hline
\end{tabular}

